\title{
ON FUNCTIONS OF SEVERAL COMPLEX VARIABLES*
}

\author{
$\mathbf{B Y}$ \\ WILLIAM F. OSGOOD
}

By means of certain very simple examples it is possible to decide a number of questions relating to analytic functions of several complex variables, which have hitherto, so far as the author is aware, remained open.

It is unsatisfactory, in stating an important theorem, not to know whether a given hypothesis is needed merely for convenience of proof, or whether the theorem would be false if it were omitted. The situation is still more annoying when it is conceivable that the theorem could be proven with about the same ease without the hypothesis, if one were only able to see more clearly.

Some of the questions to which these examples apply are of this nature. In other cases analytic phenomena of novel character are brought to light.

\section{A BRANCH MORE GENERAL THAN AN ELEMENT}

Let $x, y$, and $z$ be defined by the equations:

$$
x=f(u, v), \quad y=\phi(u, v), \quad z=\psi(u, v),
$$

where $f, \phi, \psi$ are rational functions of $u$ and $v$, all of which vanish at the origin, $(u, v)=(0,0)$; and let at least one of the two-rowed determinants in the matrix

$$
\left\|\begin{array}{lll}
\frac{\partial f}{\partial u} & \frac{\partial \phi}{\partial u} & \frac{\partial \psi}{\partial u} \\
\frac{\partial f}{\partial v} & \frac{\partial \phi}{\partial v} & \frac{\partial \psi}{\partial v}
\end{array}\right\|
$$

be not identically 0 . Then the equations (1) determine a piece of a monogenic analytic configuration-a surface - and the equation of this surface can be obtained in the form

$$
G(x, y, z)=0
$$

by eliminating $u$ and $v$. Here, $G$ denotes an irreducible polynomial vanishing at the origin.

If we replace the requirement that $f, \phi, \psi$ be rational by the hypothesis

* Presented to the Society, October 30, 1915. 
that they be merely analytic at the origin, and retain the other requirements, the equations (1) still determine a piece of a monogenic analytic configuration, and it is frequently possible to represent this piece in the neighborhood of the origin by a single equation (3), where now $G$ is analytic at the origin and vanishes there, and moreover is irreducible.* The question presents itself: Is this always possible?

This question is to be answered in the negative, as is shown by the following example.

Theorem I. The equations

$$
x=u, \quad y=u v, \quad z=u v e^{v}
$$

define a piece of a monogenic analytic configuration $G$, the coördinates of whose points do not satisfy any relation of the form

$$
\Omega(x, y, z)=0,
$$

where $\Omega$ is analytic at the origin, but does not vanish identically.

The proof is immediate. Suppose there were such a function. Let it be developed into a series of homogeneous polynomials:

and let

$$
\Omega=\Omega_{0}+\Omega_{1}+\Omega_{2}+\cdots,
$$

$$
\Omega_{n}=A_{0} x^{n}+\left(A_{1} y+B_{1} z\right) x^{n-1}+\left(A_{2} y^{2}+B_{2} y z+C_{2} z^{2}\right) x^{n-2}+\cdots .
$$

Substitute for $x, y, z$ in $\Omega$ their values from $(A)$ and develop according to ascending powers of $u$. The coefficient of $u^{n}$ will be

$$
A_{0}+\left(A_{1}+B_{1} e^{v}\right) v+\left(A_{2}+B_{2} e^{v}+C_{2} e^{2 v}\right) v^{2}+\cdots,
$$

and this coefficient must vanish for all values of $v$. Let $v=v_{1}$ be an arbitrary value of $v$. Then, in particular, (4) must vanish for the values of the argument,

$$
v=v_{1}+2 k \pi i, \quad k=0,1,2, \cdots, n .
$$

The equations thus arising from (4) may be regarded as $n+1$ linear homogeneous equations in $A_{0}$ and the parentheses. Their determinant, being the product of the differences of the numbers (5), is not 0 . Hence $A_{0}$ and each parenthesis must vanish.

Finally, if an expression of the form

$$
A+B e^{v}+C e^{2 v}+\cdots \text { to } k \text { terms }
$$

vanishes for all values of $v$, then each coefficient must vanish. For, on setting

$$
e^{v}=w,
$$

* I. e., $G$ cannot be represented as the product of two factors, each of which is analytic at the origin and vanishes there. 
this expression becomes a polynomial,

$$
A+B w+C w^{2}+\cdots
$$

This completes the proof. The example can be carried over to $n$ functions of $n-1$ parameters by setting

$$
\begin{gathered}
x_{1}=u_{1}, \quad x_{2}=u_{1} u_{2}, \quad x_{3}=u_{1} u_{2} e^{u_{2}}, \\
x_{k}=u_{1} u_{2} e^{u_{k-1}}, \quad k=4, \cdots, n .
\end{gathered}
$$

The title of this paragraph is due to the fact that Weierstrass* suggests by a distinction in terminology that he was aware of the phenomena here treated. Such equations as (1) define what he calls a branch of a monogenic analytic configuration, while an element is given by a more restricted definition.

2. ON THE IDENTICAL VANishing OF THE JACOBIAN

The following theorem is well known. $\dagger$ Let

$$
f_{i}\left(u_{1}, \cdots, u_{n}, y_{1}, \cdots, y_{m}\right) \quad(i=1, \cdots, n),
$$

be analytic in the point $(b, \beta)=\left(b_{1}, \cdots, b_{n}, \beta_{1}, \cdots, \beta_{m}\right)$, and let

$$
f_{i}\left(b_{1}, \cdots, b_{n}, \beta_{1}, \cdots, \beta_{m}\right)=a_{i} \quad(i=1, \cdots, n) .
$$

Let $\mathfrak{T}$ be an arbitrary neighborhood of the point

$$
(a, \beta)=\left(a_{1}, \cdots, a_{n}, \beta_{1}, \cdots, \beta_{m}\right) .
$$

Finally, let the jacobian

$$
\frac{\partial\left(f_{1}, \cdots, f_{n}\right)}{\partial\left(u_{1}, \cdots, u_{n}\right)}
$$

regarded as a function of the $n+m$ arguments $(u, y)$, vanish identically.

Then there exists a point $\left(a^{\prime}, \beta^{\prime}\right)$ of $\mathfrak{T}$ and a function

$$
\Omega\left(x_{1}, \cdots, x_{n}, y_{1}, \cdots, y_{m}\right)
$$

analytic at $\left(a^{\prime}, \beta^{\prime}\right)$ and such that

$$
\Omega\left(x_{1}, \cdots, x_{n}, \beta_{1}^{\prime}, \cdots, \beta_{m}^{\prime}\right) \neq 0 ;
$$

if $x_{i}$ be replaced by $f_{i}$ :

$$
x_{i}=f_{i}\left(u_{1}, \cdots, u_{n}, y_{1}, \cdots, y_{m}\right) \quad(i=1, \cdots, n),
$$

the function $\Omega$ then goes over into a function of $\left(u_{1}, \cdots, u_{n}, y_{1}, \cdots, y_{m}\right)$

* Weierstrass, in the memoir devoted to the proof of the Theta Theorem, Werke, vol. 3, p. 103, where the definition of Zweig is given, the definition of Element having just preceded.

† Cf. Peano-Genocchi, Calcolo differenziale, p. 172; Jordan, Cours d'analyse, vol. I, 2d ed., 1893, p. 86. 
which vanishes identically in these arguments:

$$
\Omega\left(f_{1}, \cdots, f_{n}, y_{1}, \cdots, y_{m}\right) \equiv 0 .
$$

Is this theorem true when the point $\left(a^{\prime}, \beta^{\prime}\right)$ is taken at $(a, \beta)$ ? The answer is given by the following theorem.

Theorem 2. a) In the case $n=2$ the above theorem is true when the point $\left(a^{\prime}, \beta^{\prime}\right)$ is taken at $(a, \beta)$.

b) When $n>2$, the theorem does not hold generally when $\left(a^{\prime}, \beta^{\prime}\right)$ is taken at $(a, \beta)$.

The first part of the theorem is due to Bliss, and was proven by him in the Princeton Colloquium of 1909, published in 1913, Chap. 2, § 12, p. 67.

To prove the second part of the theorem it is sufficient to cite one example. In the equations $(A)$ let $u$ be replaced by $u w$ :

$$
x=u w, \quad y=u v w, \quad z=u v w e^{v} .
$$

Here, it is clear that the jacobian vanishes identically, and that, moreover, the three functions are not connected by any relation of the form

$$
\Omega(x, y, z)=0,
$$

where $\Omega$ is analytic at the origin and does not vanish identically, but goes over, when the functions $(B)$ are substituted in it, into a function of $u, v, w$ which is identically zero.

For the case of $n$ arguments $u_{i}$ and $m$ parameters $y_{k}$ we may set

$$
\begin{gathered}
x_{1}=u_{1} u_{n}, \quad x_{2}=u_{1} u_{2} u_{n}, \quad x_{3}=u_{1} u_{2} u_{n} e^{u_{2}}, \\
x_{k}=u_{1} u_{2} u_{n} e^{u_{k-1}} \quad(k=4, \cdots, n) .
\end{gathered}
$$

\section{Concerning a generalization of Weierstrass's theorem of FACTORIZATION}

In the Madison Colloquium* I called attention to a generalization of Weierstrass's theorem of factorization for functions of two variables, and raised the question as to whether the theorem holds generally.

Let $F\left(u ; x_{1}, \cdots, x_{n}\right)$ be analytic at the origin and vanish there. Is it then always possible to represent $F$ in the neighborhood of the origin in the form

$$
F\left(u ; x_{1}, \cdots, x_{n}\right)=\left[A_{0} u^{m}+A_{1} u^{m-1}+\cdots+A_{m}\right] H\left(u ; x_{1}, \cdots, x_{n}\right),
$$

where $A_{k}\left(x_{1}, \cdots, x_{n}\right), k=0, \cdots, m$, is analytic at the origin, and $H\left(u ; x_{1}, \cdots, x_{n}\right)$ is analytic at the origin and does not vanish there?

That the theorem is true when $n=1$ was recognized by Risley and Macdonald. $\dagger$ When, however, $n>1$, the theorem is false, as appears from the following example.

* P. 184.

† A nnals of Mathematics, 2d ser., vol. 12 (1910), p. 73. 
Let $w=Q(z)$ be the function defined by the series*

$$
Q(z)=z+\sum_{n=2}^{\infty} \frac{z^{n !+2}}{(n !+1)(n !+2)} .
$$

This function is analytic throughout the interior of the unit circle, has the circumference of that circle as a natural boundary, and maps the above region in a one-to-one manner and conformally on a finite single-leaved region of the $w$-plane. Let $R(w)$ be the inverse function:

$$
w=Q(z), \quad z=R(w) .
$$

Then $R$ is also single-valued and analytic, the point $w=0$ lying within its domain of definition.

We proceed now to form the example required for our purpose. Consider the function

$$
z=Q\left(\frac{y}{x}\right)
$$

It is obviously a single-valued monogenic analytic function, and its domain of definition is the region

$$
0 \leqq|y|<|x| \text {. }
$$

Moreover, $z$ remains finite: $|z|<G$.

The corresponding monogenic analytic configuration is also given by the equation

$$
y-x R(z)=0 .
$$

The example is the following:

$$
F(z, x, y)=y-x R(z) \text {. }
$$

If this function permitted a factorization of the kind in question, then the configuration $(D)$ would be represented in the neighborhood of the origin by an equation of the form

$$
A_{0}(x, y) z^{m}+A_{1}(x, y) z^{m-1}+\cdots+A_{m}(x, y)=0,
$$

where $A_{k}(x, y)$ is analytic at the origin, and $A_{0}(x, y) \neq 0$.

Here, $A_{0}(x, y)$ must vanish at the origin. For otherwise we may set $A_{0}(x, y)=1$, and then either horn of the dilemma: $m>1, m=1$, leads at once to a contradiction.

Now let $\left(x_{1}, y_{1}\right)$ be chosen within an arbitrary neighborhood of the origin as a point distinct from the origin, for which $A_{0}(x, y)$ vanishes, but for which some other coefficient, $A_{k}(x, y)$, does not. It must be possible to find such a point, since otherwise all the coefficients in (7) would be divisible

\footnotetext{
* Cf. the author's Funktionentheorie, vol. 1, ch. 9, \&5.
} 
by one and the same prime factor $G(x, y)$ of $A_{0}(x, y)$; hence $(E)$ must also be divisible by $G$, and this is evidently not the case.

Let $\left(x_{0}, y_{0}\right)$ be a point of the domain (6), lying in the neighborhood of the origin, and let $A_{0}\left(x_{0}, y_{0}\right) \neq 0$. Let the discriminant $D(x, y)$ of (7) also be different from 0 in this point. Then $z_{0}=Q\left(y_{0} / x_{0}\right)$ will be one of the roots of (7).

We now proceed to let the point $(x, y)$ move along a path emanating from $\left(x_{0}, y_{0}\right)$ and leading to $\left(x_{1}, y_{1}\right)$, but passing through no point in which either $A_{0}(x, y)$ or $D(x, y)$ vanishes. The function $z$ defined in the neighborhood of $\left(x_{0}, y_{0}\right)$ by $(C)$ must admit an analytic continuation along this path, since this is true for the same function, regarded as a root of (7).

On the other hand, at least one of the roots of (7) becomes infinite as $(x, y)$ approaches $\left(x_{1}, y_{1}\right)$, and we can choose the path so that the above function $z$ will be carried over into such a root. For, even though (7) may be reducible, still the function $(C)$ satisfies an irreducible equation of the same type as (7), the left-hand side of the equation being a factor of the left-hand side of (7); and we may choose $\left(x_{1}, y_{1}\right)$ with reference to the irreducible equation. Here is a contradiction, and thus the following theorem is established.

Theorem 3. When $n=1$, the question proposed at the beginning of the paragraph is to be answered in the affirmative; when $n>1$, in the negative.

A further example which illustrates the same point, but in which the whole monogenic analytic configuration lies in the finite region is the following:

(F) $\quad x=u, \quad y=u v, \quad z=Q(u)+Q(v), \quad|u|<1, \quad|v|<1$; or:

or still again:

$$
z=Q(x)+Q\left(\frac{y}{x}\right), \quad 0 \leqq|y|<|x|<1 ;
$$

$$
y=x R[z-Q(x)] .
$$

In the last case, $|x|<1, Q(x)$ remains finite, and $z$ must be so chosen that the point $z-Q(x)$ lies in the domain of definition of the function $R$. Thus $y$ also remains finite.

\section{Geometric interpretation. A fURther example}

Consider the function

$$
z=\frac{y}{x}
$$

in the neighborhood of the origin, $(x, y)=(0,0)$. The function is analytic at all points there save those for which $x=0$. But its value does not remain finite.

In fact, we can picture the function to ourselves in a way by considering 
the real surface corresponding to real values of $x$ and $y$. This surface is spread out over the neighborhood of the origin, the line $x=0$ in the $(x, y)$ plane being excepted, and is generated by a line

$$
y=z_{0} x, \quad z=z_{0},
$$

which always cuts the $z$-axis, is parallel to the $(x, y)$-plane, and rotates as its point of intersection with the $z$-axis advances. The one direction in the $(x, y)$-plane to which this line never becomes parallel is that of the $y$-axis; and in order to make a small angle with that direction, it has to recede far.

On the other hand, consider the function $(C)$ :

$$
z=Q\left(\frac{y}{x}\right)
$$

and set over against it the surface

$$
y=x R(z) .
$$

Think of this surface as generated by the moving line:

$$
y=R\left(z_{0}\right) x, \quad z=z_{0} .
$$

This is a surface of precisely the same character as the one generated by the moving line $(g)$, the difference being merely that the rate at which the moving line rotates as its point of intersection with the $z$-axis travels along that line is different; and, moreover, that this point does not go so far, being restricted to a finite field.

When we project the surface $\left(H^{\prime}\right)$ on the $(x, y)$-plane, we do not get approximately the whole neighborhood of the origin, but only so much, in the domain of reals, as lies within a certain angle.

This fact is suggestive for the complex case. The analogue of the angular region just referred to is the portion of the four-dimensional space defined by the relations (6).

That which the two examples $(G)$ and $(H)$ have in common is this, that when we consider the part of the monogenic analytic configuration which lies in the (complex) three-dimensional neighborhood of the origin $(x, y, z)$ $=(0,0,0)$, we obtain each time a piece of an analytic surface,

$$
y=\omega(x, z),
$$

where $\omega$ is analytic at the origin and vanishes there, namely

$$
\omega(x, z)=x z \quad \text { resp. } \quad \omega(x, z)=x R(z) .
$$

That in which the two examples differ is this. When the equation $(K)$ is solved for $z$, we get in the one case a function $(G)$ having a non-essential singularity at the origin, $(x, y)=(0,0)$. In the other case, the solution 
of $(K)$ for $z$ yields a function $(H)$ having at the origin a singularity for which the algebraic functions yield no prototype. The origin lies on the boundary of a lacunary space; it is a point of a natural boundary of a single-valued monogenic function of $x$ and $y$.

Thus we have been led by the geometric picture to an example which justifies the following theorem.

TheOREm 4. Let $G(x, y, z)$ be a function analytic at the origin and vanishing there, and let $G$ be irreducible. Furthermore, let $G$ actually depend on $z$. Then the equation $G(x, y, z)=0$, when solved for $z$ may yield a single-valued monogenic analytic function which has at the origin $(x, y)=(0,0)$ a singularity for which algebraic and algebroid functions yield no prototype.

In fact, the origin may be a point of a natural boundary, the neighborhood of the origin consisting in part of a lacunary space.

Two remarks. - First, we have obtained a property of analytic functions in two variables which is distinctly unlike what we know to be the case for such functions in a single variable. There, if $y=f(x)$ is a function analytic at a point $x=a$ or having there at most a branch-point or pole (the most general singularity of which algebraic or algebroid functions of one variable are capable), and if $f(x)$ actually depends on $x$ (i. e., is not a constant) then the inverse function $x=\phi(y)$ can have at the corresponding point at most a singularity of algebraic type.

The second remark is this. While it is easy to form real functions of two real variables which are analytic in disconnected regions of the $(x, y)$-plane, but are, in the complex domain, merely branches of one and the same monogenic analytic function, $-\log \sin (x+y)$ is, for example, such a function,still, the monogenic functions involved are apt to be multiple-valued.

In the functions $\left(F^{\prime}\right)$ and $(H)$ we have single-valued monogenic analytic functions exhibiting this property.

If it be desired to have the two domains of definition finite and without even a boundary point in common, the following function will serve the purpose:

$$
\begin{gathered}
F(x, y)=Q(x)+Q\left(\frac{1}{2 x}\right)+Q\left(\frac{y}{x}\right), \\
\frac{1}{2}<|x|<1, \quad 0 \leqq|y|<|x| .
\end{gathered}
$$

Harvard University,

June 10, 1915 